\title{
Successful laparoscopic management of a rare complication after embryo transfer: ovarian pregnancy. A case report and up-to-date literature review
}

\author{
Turgut Aydin ${ }^{1}$, Burak Yucel ${ }^{1}$, Huseyin Aksoy², Suheyla Ekemen ${ }^{3}$ \\ ${ }^{1}$ Assisted Reproduction Unit, Acibadem Hospital, Kayseri, Turkey \\ 2Department of Obstetrics and Gynecology, Military Hospital, Kayseri, Turkey \\ ${ }^{3}$ Department of Pathology, Acibadem Aile Hospital, Istanbul, Turkey
}

Videosurgery Miniinv 2015; 10 (4): 574-579

DOI: $10.5114 /$ wiitm.2015.55893

\begin{abstract}
Ovarian pregnancy (OP) after embryo transfer is very rare. Due to the rarity and the asymptomatic nature, there are still difficulties in diagnosis and treatment. The traditional operative treatment for OP has been oophorectomy. However, the desire to maintain reproductive capability and improvements in laparoscopy have more recently led to conservative laparoscopic techniques. This rare complication could be diagnosed early and managed by a conservative laparoscopic approach. Here we present a survey of the literature and a case of successful laparoscopic management of ovarian pregnancy after intracytoplasmic sperm injection and embryo transfer. The current case is the first case in the literature in which ovarian pregnancy occurred after a single embryo transfer. We also summarize the literature about management of ovarian pregnancy after embryo transfer.
\end{abstract}

Key words: ovarian pregnancy, laparoscopic surgery, fertilization in vitro, infertility.

\section{Introduction}

Approximately one percent of all pregnancies are ectopic. An increased incidence of ectopic pregnancies (5\%) after intracytoplasmic sperm injection - embryo transfer (ICSI-ET) is a well-known phenomenon. Ovarian ectopic pregnancy (ovarian pregnancy - OP) is a rare phenomenon following natural conception (3\% of all ectopic pregnancies) or ICSI-ET (0.27\% per clinical pregnancy) [1].

The traditional operative treatment for OP has been oophorectomy. However, the desire to maintain reproductive capability and improvements in laparoscopy have more recently led to conservative laparoscopic techniques. Conservative management methods are gaining more importance in infertile patients who have received assisted reproduction treatments [2].

Here, we present a case of OP after ICSI-ET, and an up-to-date literature review. This case differs from previously presented cases. In the literature, all OP cases after ICSI-ET occurred with the transfer of multiple (2 or more) embryos. The OP occurred after a single ET in our case.

\section{Case report}

She was 31 years and primary infertile for five years. In her history, she noted that she had an operation of vaginal septum resection 4 years before (Table I). Because of male factor infertility (non-obstructive azoospermia), the couple was taken into 
Table I. Properties of the case

\begin{tabular}{|cccccccc|}
\hline Age & Indication & Attempt & $\begin{array}{c}\text { Transfer } \\
\text { day }\end{array}$ & $\begin{array}{c}\text { Number of } \\
\text { transferred } \\
\text { embryos }\end{array}$ & $\begin{array}{c}\text { Implantation } \\
\text { site }\end{array}$ & Surgery & Prognosis \\
\hline 31 & $\begin{array}{c}\text { Male factor } \\
\text { (non-obstructive } \\
\text { azoospermia) }\end{array}$ & 2. & $5^{\text {th }}$ & 1 & Right & $\begin{array}{c}\text { Laparoscopic re- } \\
\text { moval of the mass }\end{array}$ & $\begin{array}{c}\text { After one more } \\
\text { attempt with frozen } \\
\text { embryo transfer, } \\
\text { pregnant }\end{array}$ \\
\hline
\end{tabular}

the assisted reproduction program. They previously had one ICSI cycle without conception in a different in vitro fertilization (IVF) center. Histopathological diagnosis was hypospermatogenesis. 200 IU of recombinant follicle-stimulating hormone (FSH) (Gonal-F; Merck Serono S.p.A, Modugna, Ba, Italy) combined with a gonadotrophin-releasing hormone $(\mathrm{GnRH})$ antagonist protocol (Cetrorelix; Merck Serono, Halle, Germany) were administered daily for controlled ovarian stimulation. Oocyte triggering was achieved by the administration of human chorionic gonadotropin (hCG) (Pregnyl; Organon S.p.A, Roma, Italy). Eight oocytes were retrieved. Seven of them were mature (metaphase II: M2) and injected. Four embryos cleaved to day three and then three 8-cell embryos cleaved to the blastocyst stage. A single expanded blastocyst was transferred at day five using a soft catheter (Wallace Sure View Embryo Replacement Catheter; Smith Medical, Hythe, Kent, UK) and the remaining two embryos were frozen. Vaginal progesterone gel (Crinone; Merck Serono, Bedfordshire, United Kingdom) was given for luteal support.

Serum $\beta$-hCG on the $10^{\text {th }}$ day of embryo transfer was $39 \mathrm{mIU} / \mathrm{ml}$. After 2 days, serum $\beta$-hCG increased to $83 \mathrm{mlU} / \mathrm{ml}$. All findings were normal. She was admitted to our hospital with lower abdominal pain 2 weeks later. She was pale, but the vital signs were stable. Pelvic examination revealed a tender right adnexal mass. Serum $\beta$-hCG was $1.634 \mathrm{mlU} / \mathrm{ml}$. Transvaginal sonography showed that the uterine cavity was empty; significant free fluid in the pouch of Douglas and right ovary had a hypo-echoic area of $2 \times 2 \mathrm{~cm}$. In addition, Doppler sonography revealed increased peripheral vascularity giving a ring-of-fire appearance around the hypo-echoic area.

Emergency laparoscopy was performed with the pre-diagnosis of ectopic pregnancy. In the overview, hemorrhage from the ruptured ectopic pregnancy was identified; there was approximately $500 \mathrm{ml}$ of bloody, clotted fluid in the abdomen. In the background, a soft, slightly enlarged uterus was found.
The left and right fallopian tubes were normal without dilatation or bleeding from the fimbrial end. The left ovary showed a fresh corpus luteum cyst without bleeding. The approximately $2 \mathrm{~cm}$ well-defined gestational sac, implanted on the right ovary, was actively bleeding (Photo 1). The product of conception was bluntly prepared and enucleated from the orthotropic ovarian tissue and removed from the ovary using microscissors and spoon forceps. After complete separation of the trophoblast from the right ovary, it could be easily removed from the abdominal cavity through a $10 \mathrm{~cm}$ trocar in the midline. Adequate hemostasis on the ovarian tissue was maintained using bipolar coagulation. Uterine curettage was also performed and showed proliferative endometrium free of chorionic villi. Histopathologic examination of the tissues removed from the ovary showed signs of diffuse bleeding alongside chorion villi, decidual cells and decidual changed stroma with normal ovarian cortical tissue in the outer margins (Photo 2).

She was discharged a day after the operation. Post-operative recovery was excellent. Follow-up quantitative serum $\beta$-hCG levels declined appropriately and reached an undetectable level in 2 weeks. Three months later, she had thawed single embryo transfer; she conceived and has an ongoing 22 weeks pregnancy.

\section{Discussion}

Ovarian pregnancy is one of the rarest forms of ectopic pregnancies with a reported incidence of from $1 / 7,000$ to $1 / 60,000$ pregnancies and accounts for about $1-3 \%$ of all extra-uterine pregnancies [3]. On the other hand, OP after ICSI-ET is even rarer, and just a few cases have been reported in the literature. For this review, we performed a PubMed search, using the keywords "Primary ovarian ectopic pregnancy", "ovarian pregnancy", "intracytoplasmic sperm injection embryo transfer" and "ICSI-ET" for the last 20 years. It revealed 13 papers and 15 cases in the literature from 1994 to the present (Table II). 


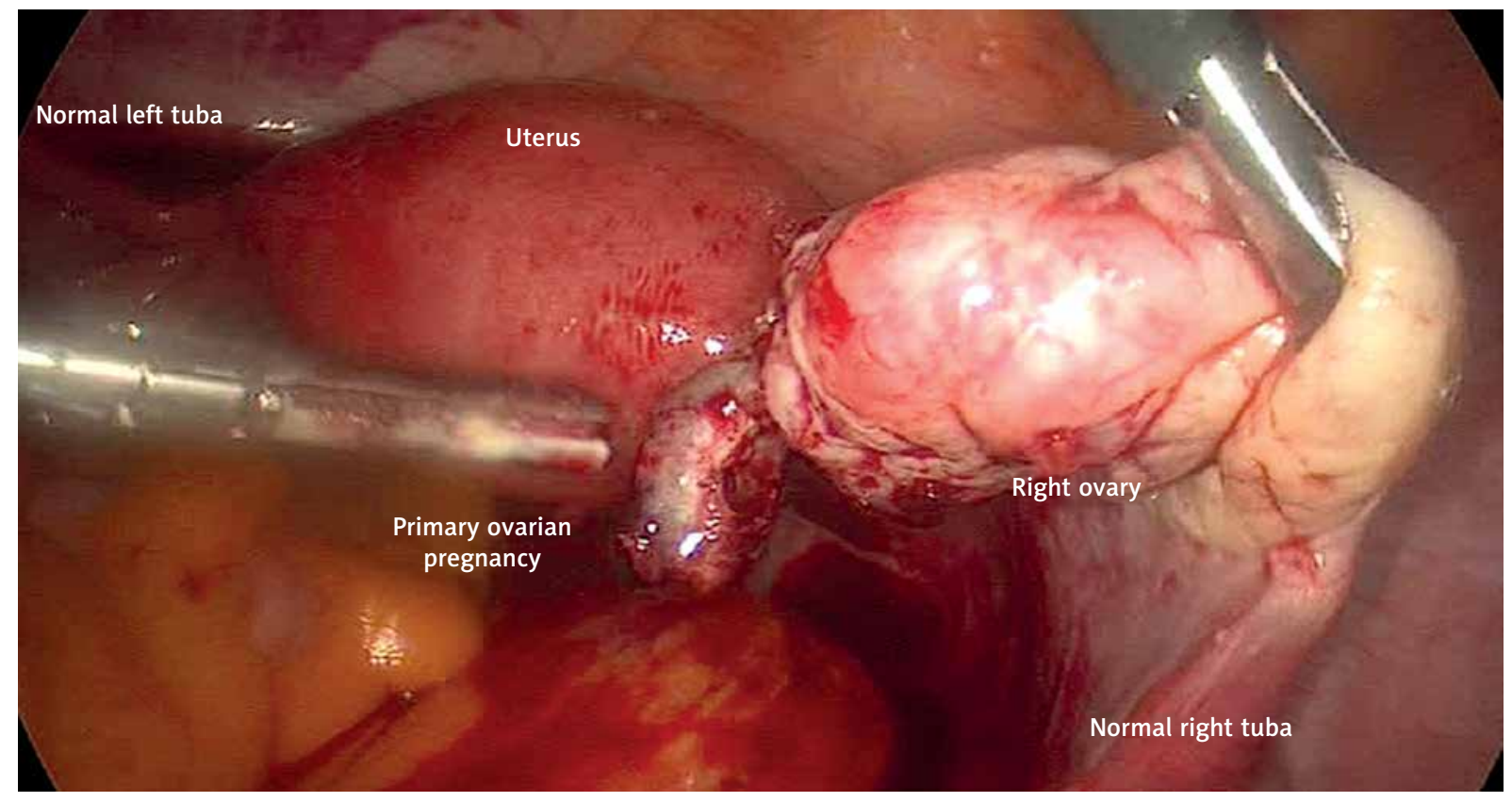

Photo 1. Intraoperative view of pelvis

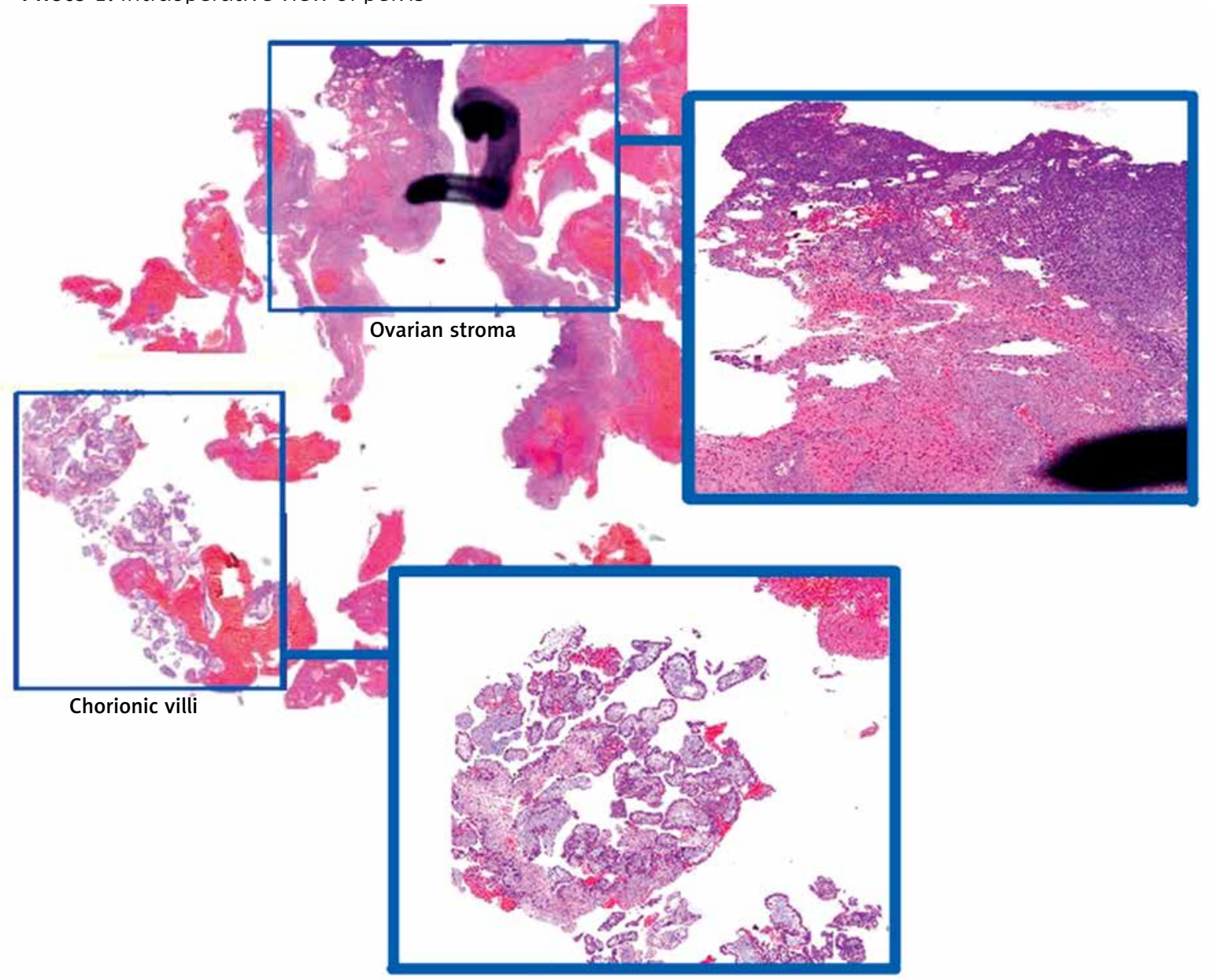

Photo 2. Histopathologic view of ovarian pregnancy 


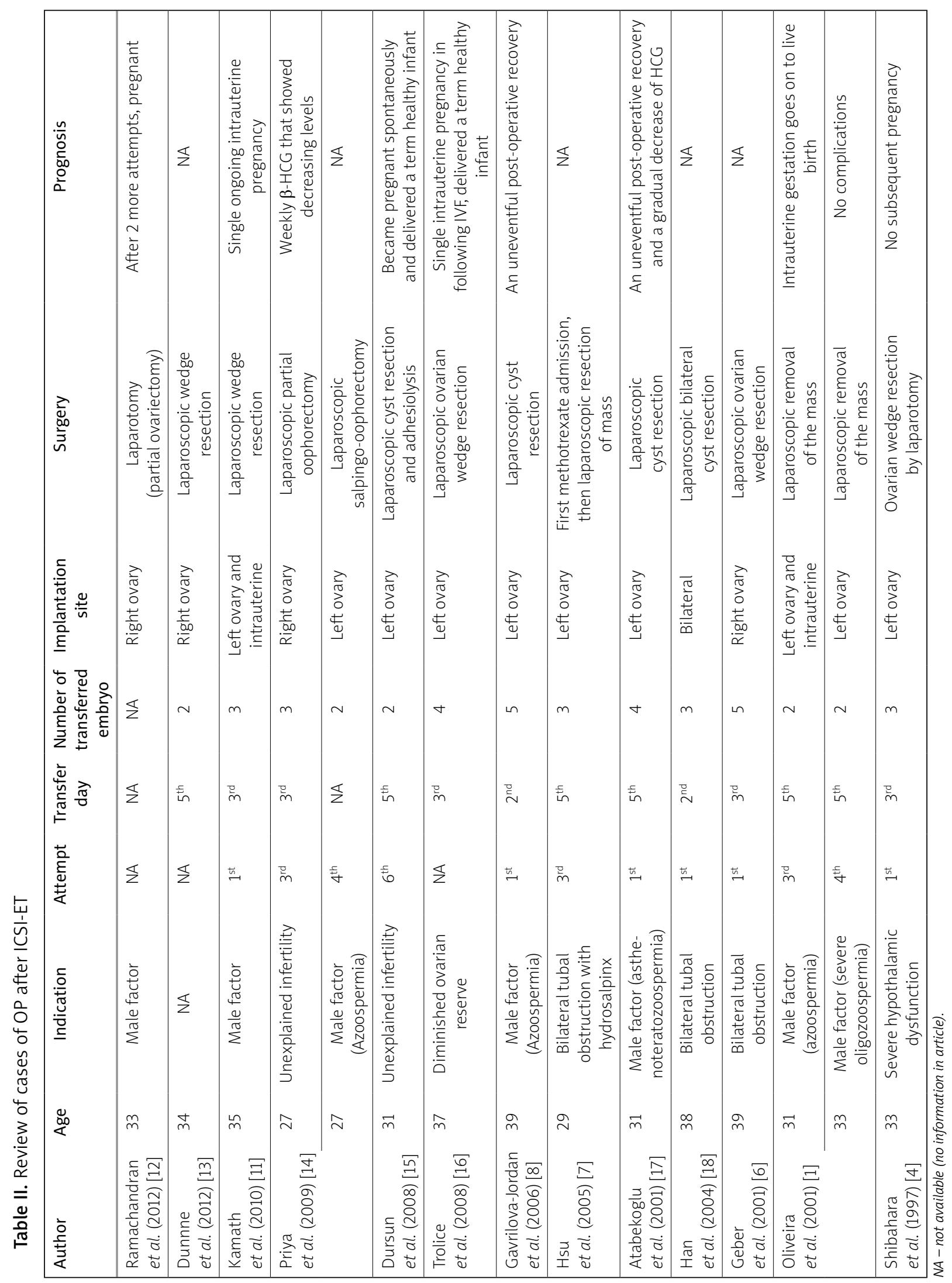


Ages of patients were between 27 and 39 years. This is consistent with the age of women in whom ICSI-ET is indicated. Our patient was 31 years old. Male factor infertility was the most frequent indication for ICSI-ET treatment in 7 of 15 cases. Since there is no relationship between male factor infertility and implantation of embryo, this excess might be incidental. In our case, the indication for assisted reproduction was also severe male factor infertility (non-obstructive azoospermia). Histopathological diagnosis was hypospermatogenesis.

The exact etiology of an OP after IVF-ET is not known, although reverse migration of the embryo have been postulated [4]. Some factors in ET could cause this unusual event: large volume and high pressure of culture medium injected during embryo transfer, difficult ET, manipulation with tissue forceps. Moreover, it is also speculated that high estrogen levels stimulate uterine contractions and can increase the risk. In addition, the risk appears to be inversely correlated with decreasing distance of embryo placement to the uterine fundus [5] at commencement of down-regulation.

Interestingly, OP after ICSI-ET was defined in a case with absent tubes. In addition, abdominal pregnancies after ICSI-ET in women with previous bilateral salpingectomy have been described [6]. However, the cause of ectopic implantation of embryos in the women who had had a bilateral salpingectomy in both of those studies was not well defined. There can be suggested another mechanism in which corneal fistulae might be the most probable explanation of the cause of these 2 cases.

Various anatomical defects, such as vaginal septum, uterine septum, and arcuate uterus, were presented in cases of the reviewed literature. Our patient had a history of vaginal septum resection. In contrast to women with tubal pregnancies, wellknown risk factors such as pelvic inflammatory disease and prior pelvic surgical procedures might not play a role in the etiology of ovarian pregnancy. The mechanism is not about blockage of embryo migration, but rather reverse migration of embryos from the uterus to the ovary [7]. Whereas in 3 cases of the reviewed literature there were histories of locked tubes or hydrosalpinx, in 1 case there was a history of tubal re-anastomosis.

Depending on the past reports, blastocyst transfer and multiple embryo transfers (three or more) seem to be the most important predisposing factors for OP after ICSI-ET. The mean number of transferred embryos in the reviewed literature was 3.3. Oliveira et al. [1] also confirmed that blastocyst embryo transfer on the fifth day might be an underlying mechanism for OP. More embryos mean more chance to implant on undesirable areas. However, as our case showed, OP can occur after single embryo transfer.

Diagnosis of OP is difficult because of the rarity and the asymptomatic nature before rupture. The variability of clinical signs and symptoms might be initially misleading. Differential diagnosis of hemorrhagic corpus luteum or a ruptured lutein cyst can be easily missed. Initial serum $\beta$-hCG levels might not be decisive. Abnormally low and slow-rising serum $\beta$-hCG aids in early recognition of abnormal implantation, but it is not specific to ovarian pregnancy. Ultrasonographic signs of ovarian pregnancy might be obscured by multiple corpora lutea cysts after hyperstimulation and egg retrieval in a standard ICSI-ET cycle. The decisive ultrasonographic characteristics are: (i) the visualization of gestational sac structures, (ii) peripheral vascularity giving the classic ring-offire appearance in Doppler ultrasonography as in our case or (iii) the presence of fetal heart beat within the ovary [8]. The advice that must be underlined is that a high degree of suspicion of an ovarian ectopic pregnancy is required.

The first operative steps are the same as for tubal pregnancy. Once the ectopic pregnancy has been localized and bleeding is under control, an operative plan is decided upon. Earlier, laparotomic wedge resections were performed as a traditional method; however, improvements in laparoscopic techniques and advances in surgical experience have led to surgeons performing laparoscopic enucleation of the OP. Laparoscopic enucleation of the gestational product is the gentlest type of operation. By enucleating the gestational sac bluntly from the ovary, the surrounding ovarian tissue is protected to the greatest possible extent in these patients suffering from infertility. Careful surgical extraction of the trophoblastic tissue from the place of implantation under optical magnification in laparoscopy is an essential precondition [2]. In the literature, all cases with $O P$ after ICSI-ET, except two, were successfully treated with laparoscopic resections. The remaining 2 cases were treated by laparotomies. We also performed laparoscopic enucleation in our case.

Operative laparoscopy has the benefit of reduced morbidity, reduced hospitalization and rapid recov- 
ery of the patient. In addition, it has an advantage of reduced postoperative adhesions compared to laparotomy. Laparoscopy is the gold standard in the diagnosis and treatment of an OP [2]. Conservative treatments should be an appropriate option for these infertile patients and can be performed with early diagnoses. Successful treatment of ovarian pregnancy with methotrexate has also been reported [9]; however, limited literature is available on the subject and factors predicting success of treatment are not clearly defined.

Since a certain diagnosis may not be established during the operation, histopathological confirmation is an important point for OP after ICSI-ET. In some cases, differential diagnosis of hemorrhagic corpus luteum or a ruptured lutein cyst can only be performed by histopathological examination. Spiegelberg suggested four criteria to distinguish a primary ovarian pregnancy pathologically. These criteria can be summarized as follows: (i) the fallopian tube with its fimbria should be intact and separate from the ovary, (ii) the gestational sac should occupy the normal position of the ovary, (iii) the gestational sac should be connected to the uterus by the ovarian ligament, and (iv) ovarian tissue must be present in the specimen attached to the gestational sac [10]. Our case fulfills all these criteria.

Reported patients in the literature had uneventful postoperative recoveries. The course after conservative treatment with laparoscopy is excellent. Most of patients in the literature conceived after surgery spontaneously or with repeated ICSI-ET. Likewise, our patient conceived with thawed single embryo transfer 3 months later. Moreover, in two heterotopic pregnancies, intrauterine pregnancies had gone on to be healthy after resection of ovarian pregnancies by laparoscopy $[1,11]$.

\section{Conclusions}

Awareness of the possibility of OP after ICSI-ET is the most important step of early diagnosis and treatment. Laparoscopic enucleation should be safely performed in experienced hands for treatment of this very rare complication, especially for women who wish to preserve their fertility potential.

\section{Conflict of interest}

The authors declare no conflict of interest.

\section{References}

1. Oliveira FG, Abdelmassih V, Costa a L, et al. Rare association of ovarian implantation site for patients with heterotopic and with primary ectopic pregnancies after ICSI and blastocyst transfer. Hum Reprod 2001; 16: 2227-9.

2. Alkatout I. Ectopic pregnancy. In: Practical manual for laparoscopic \& hysteroscopic gynecological surgery. Jaaype Brother Medical Publishers 2013; 169-89.

3. Bontis J, Grimbizis G, Tarlatzis BC, et al. Intrafollicular ovarian pregnancy after ovulation induction/intrauterine insemination: pathophysiological aspects and diagnostic problems. Hum Reprod 1997; 12: 376-8.

4. Shibahara H, Funabiki M, Shiotani T, et al. A case of primary ovarian pregnancy after in vitro fertilization and embryo transfer. J Assist Reprod Genet 1997; 14: 63-4.

5. Lesny P, Killick SR, Robinson J, et al. Junctional zone contractions and embryo transfer: is it safe to use a tenaculum? Hum Reprod 1999; 14: 2367-70.

6. Geber S, Barroso R, Pereira D, Sampaio M. Ovarian pregnancy after IVF - ET in a patient with absent tube. J Assist Reprod Genet 2001; 18: 665-7.

7. Hsu CC, Yang TT, Hsu CT. Ovarian pregnancy resulting from cornual fistulae in a woman who had undergone bilateral salpingectomy. Fertil Steril 2005; 83: 205-7.

8. Gavrilova-Jordan L, Tatpati L, Famuyide A. Primary ovarian pregnancy after donor embryo transfer: early diagnosis and laparoscopic treatment. JSLS 2006; 10: 70-3.

9. Shamma FN, Schwartz LB. Primary ovarian pregnancy successfully treated with methotrexate. Am J Obstet Gynecol 1992; 167: $1307-8$

10. Spiegelberg O. Casuistry in ovarian pregnancy. Arch Gynecol Surv 1878; 13: 73-9.

11. Kamath MS, Aleyamma TK, Muthukumar K, et al. A rare case report: ovarian heterotopic pregnancy after in vitro fertilization. Fertil Steril 2010; 94: 1910.e9-11.

12. Ramachandran A, Sharma S, Pratap K, et al. Ovarian pregnancy following intracytoplasmic sperm injection and Embryo Transfer: a case report. Case Rep Obstet Gynecol 2012; 2012: 389107.

13. Dunne C, Havelock JC. Ovarian ectopic pregnancy after in vitro fertilization. J Obstet Gynaecol Can 2012; 34: 409.

14. Priya S, Kamala S, Gunjan S. Two interesting cases of ovarian pregnancy after in vitro fertilization-embryo transfer and its successful laparoscopic management. Fertil Steril 2009; 92: 394.e17-9.

15. Dursun P, Gultekin M, Zeyneloglu HB. Ovarian ectopic pregnancy after ICSI-ET: a case report and literature review. Arch Gynecol Obstet 2008; 278: 191-3.

16. Trolice MP, Dozier SC. Ovarian pregnancy after in vitro fertilization. Fertil Steril 2008; 89: 436-7.

17. Atabekoglu C, Berker B, Dunder I. Ovarian ectopic pregnancy after intracytoplasmic sperm injection. Eur J Obstet Gynecol Reprod Biol 2004; 112: 104-6.

18. Han M, Kim J, Kim H, et al. Bilateral ovarian pregnancy after in vitro fertilization and embryo transfer in a patient with tubal factor infertility. J Assist Reprod Genet 2004; 21: 181-3.

Received: 17.08.2015, accepted: 28.09.2015. 